\title{
La lectura de Fitting the Pattern en el aula'
}

\section{Reading Fitting the Pattern by Christine Wilks in the Classroom}

\author{
YOLANDA DE GREGORIO ROBLEDO \\ Universidad de Cádiz \\ España \\ Yolanda.degregorio@uca.es
}

(Recibido: 03/12/20I8; aceptado: $07 / 05 / 2019$ )

Resumen. La aparición de los dispositivos electrónicos y las nuevas obras creadas para ellos tanto en línea como en aplicaciones, ha abierto un campo importante de estudio para la Teoría de la Literatura desde la que estudiamos en este trabajo la lectura digital. En concreto, en el presente artículo identificamos algunos de los elementos que muestran que leer una obra de literatura digital sea una actividad diferente a leer un texto impreso, así como las dificultades que conlleva, retándonos con ello a formar a nuestros estudiantes universitarios en nuevas destrezas y códigos. Además, tras señalar los rasgos generales y las claves de lectura de la obra de narrativa digital Fitting the Pattern de Christine Wilks, proponemos una aplicación práctica de lectura de esta creación como herramienta para el aprendizaje, no solo de la lectura digital sino también del inglés y de estudios culturales para las alumnas y alumnos del grado de Estudios Ingleses.

Palabras clave: literatura digital; lectura digital; teoría de la literatura; narrativa; didáctica de la lectura.
Abstract. The emergence of new electronic devices and apps with new literature pieces has opened up new possibilities in the field of Literature Theory. This paper studies digital reading. In particular, this research identifies some elements showing that reading a digital work of literature differs from a printed one. This challenges teachers to provide students with new skills and codes. To approach this, this paper explores the main characteristics of the narrative piece Fitting the Pattern, written by Christine Wilks. Finally, this paper proposes a new reading practice application using the mentioned narrative piece. Therefore this learning tool is intended for digital reading, English Second Language learning, and cultural studies among undergraduate students of English Literature.

Keywords: electronic literature; digital reading; Theory of Literature; narrative; teaching reading.

\footnotetext{
I Para citar este artículo: Gregorio Robledo, Yolanda (2O2O). La lectura de Fitting the Pattern en el aula. Alabe 21. 


\section{Dificultades de la lectura digital}

Hace unos años cuando usábamos el metro cada día para desplazarnos, veíamos cómo un gran número de usuarios llevaban un libro en la mano o un libro electrónico para hacer más entretenido su viaje. Hoy, los móviles y la lectura en ellos es lo que caracteriza a los viajeros. Cuando entramos en un aula, son estos dispositivos los que suelen estar en un lugar visible y de fácil acceso para nuestros estudiantes, y para nosotros. Luego, si la lectura en dispositivos digitales (en todas sus variedades) forman parte de lo cotidiano, ¿podríamos utilizarlo en la lectura en nuestro aula? Y ¿se podría utilizar la literatura de vanguardia o experimental, aquella creada exprofeso para los ordenadores o tabletas, denominada literatura digital o electrónica, para realizar actividades de lectura? Este artículo pretende desde la Teoría de la Literatura, responder afirmativamente a las preguntas anteriores. También pretende apuntar los elementos que hacen que la lectura de obras de literatura digital sea diferente del resto y concluye con una aplicación práctica de una lectura en el aula, en concreto de la obra de narrativa digital Fitting the Pattern'2.

La lectura puede resultar, en ocasiones, una tarea poco sencilla. Si nos adentramos en la lectura de obras digitales, su dificultad aumenta ya que a la capacidad lectora hay que añadirle un entrenamiento, una dinámica y un dominio múltiple de códigos y destrezas (Morales Sánchez 20ı8, p. I3). Pero antes de señalar sus dificultades, ¿a qué nos referimos con lectura digital? Encontramos una definición en el Diccionario Digital de Nuevas Formas de Lectura y Escritura (DINle):

La lectura digital es la que se desarrolla en cualquier tipo de pantalla mediante el uso de un programa que permite visualizar correctamente los contenidos, que pueden ser textuales, iconográficos o audiovisuales. Se trata de un tipo de lectura que se desarrolla en diferentes tipos de dispositivos con alternancias según los textos y las prestaciones que ofrecen los mismos. Tablet, e-reader o dispositivos dedicados, que emplean la tinta electrónica, Smarphones y ordenadores tanto portátiles como de mesa son los principales espacios en los que se desarrolla ésta.

\section{(Cordón García, 2018 en DINle)}

Aunque en la definición anterior se hace referencia a la lectura en cualquier dispositivo digital, en el presente artículo, nos centramos únicamente en la lectura de obras de lectura digital, es decir en aquellas obras nacidas digitales, digitales desde su origen, creadas en un ordenador (Hayles 2008, p. 3), aquellas que no pueden leerse en un ereader ni en papel ya que no podemos imprimirlas, si lo hiciéramos se perderían sus componentes. Pero el resto de la cita nos explica la actividad de la que hablamos para caminar

\footnotetext{
${ }^{2}$ Esta contribución forma parte de la línea de investigación y desarrollo del grupo Lecrired.
} 
hacia el acto de leer ese tipo de literatura. Jessica Pressman (20I4, p. 46) señala cómo ante estas obras el lector debe cambiar y redireccionar su atención para poder absorber el argumento, podemos añadir además que se ha de tener algún tipo de participación para poder ir avanzando en la obra como pinchar con el ratón en algún espacio, superar alguna prueba o simular alguna acción. Con ello se constata que el lector ha de comprender la propuesta que el autor hace para poder adentrarse en una lectura que no es tan lógica como la que encontramos en un libro en el que los párrafos siguen a las páginas, etc., mientras que en la digital, a veces habrá que encontrar las palabras, otras solo se escucharán o habrá que realizar alguna acción y leer simultáneamente, etc. Por todo esto se puede afirmar (Goicochea 2OII, p. I), que estamos ante nuevos rituales de lectura en pantalla y "Now it is time to rethink what reading is and how it Works in the rich mixtures of words and images, sounds and animations, graphics and letters that constitute the environments of twenty-first-literacies" (Hayles 2OIO, p. 78).

$\mathrm{Al}$ acercarnos a la lectura digital, constatamos en seguida que el lector es un lector itinerante (Morales Sánchez 20IO, p. 389), con el que con su acto de leer aparecen nuevos conceptos como el de navegar (en inglés encontramos: browsing, surfing y navigating) (Gervais 2007, p. I86), elegir, explorar. Se enfrenta a "un reto conceptual y tecnológico, en el que no solo debe ser capaz de leer y comprender lo que lee, sino también a aprender cómo se lee y qué se esconde tras la lectura” (Morales Sánchez 2or8, p. 9). Así la lectura como actividad, relaciona los actos de la manipulación, la comprensión y la interpretación complementándose entre ellas en nuestra progresión a través de los textos (Gervais 2007, p. I86). Todos estos aspectos pueden provocar que el lector se desoriente en los laberintos que se le propone, entre las imágenes, el texto, el sonido... y habrá que ir dándole estrategias y orientándose para que pueda sumergirse en la obra. Para ello, es importante que sea consciente que cada elemento de la pantalla está al servicio de la narración y la estética y que puede materializarse a través de diferentes textualidades y con diferentes grados de implicación e intervención en el texto. Como consecuencia de todo ello, hay una mayor necesidad de adoptar un rol más activo y crítico en la interpretación de las obras con las que hemos de formar (Valero, M. J., Vázquez, B. y Cassany, D. 2OI5, p. 8). Y, para abordar todos estos retos debe formalizarse una competencia literaria sólida que facilite tanto la estimulación del lector como el tratamiento didáctico de la literatura (Torres-Toukoumidis, A., Romero-Rodríguez, L., Pérez-Rodríguez, M. A., \& Björk, S. 20I6, p. 39) y recordar que el medio no enseña a leer ningún tipo de literatura, ni la de papel ni la digital. Es más, los lectores más competentes en los nuevos medios serán aquellos que ya lo eran en los tradicionales (Morales Sánchez 20I5, p. 68). 


\section{Rasgos generales de Fitting the Pattern: Or Being a Dressmaker's Daughter ${ }^{3}$}

La Electronic Literature Organization en su intento de preservar y de formalizar un corpus de obras de literatura digital ha ido publicado en Internet tres colecciones abiertas a todo el público de este tipo de obras en diferentes idiomas. Entre las que encontramos en el segundo volumen publicado en el año 2OII es la obra de Christine Wilks.

La obra de narrativa digital creada en el 2008 y modificada en el 2009 por Christine Wilks es una obra interactiva, animada, producida en Flash (programa que ya no se comercializa pero con el que podemos seguir visualizando la obra si lo activamos) que como ella misma describía (Mencia, M y Husárová 2015, p. 37), es una memoria en piezas, en fragmentos. Son recuerdos a los que llegar cortándolos, cosiéndolos, prendiéndoles con alfileres, descosiendo el pasado, explorando la relación que tenía con su madre que era costurera. El crear esta memoria así se debe, según Wilks, a que los misterios de la vida no suelen estar dominados por la lógica o por un proceso lineal de deducción, sino que a él se llega a través de respuestas a ideas, a intuiciones, al azar, a fragmentos que nos surgen en nuestra mente. Y, solo con el tiempo se llega a construir la idea, pieza a pieza, cambiando las fechas, mezclando hasta llegar a ver el patrón que emerge. Señala que si nuestras memorias no son lineares, ¿por qué resistirse a ello? Pues no tienen un proceso lineal sino que son caóticas, desordenadas, fragmentadas, cíclicas, variables, etc. Esta experiencia es la que se intenta reproducir en Fitting the Pattern. Or being a dressmarker's daughter. Por tanto es una memoria en piezas que puede ser explorada por el lector.

El diseño visual se basa en la estética de patrones de costura. Los cursores están diseñados como utensilios de costura y con ellos se fusiona el proceso interactivo con el mundo narrativo pues el lector se implica activamente al cortar, descoser, coser, etc. Para la autora, las herramientas tienen varias funciones, son botones de la barra de navegación; cursores personalizados; y son, como ya se ha explicado, elementos activos de la narrativa, como personajes animados, jugando literalmente su parte en la historia mientras que simultáneamente activa la experiencia de lectura. Más aún, cada herramienta activa un aspecto diferente de la historia: cortar con las tijeras desencadena recuerdos con emociones negativas, mientras que el coser con la máquina muestra sentimientos positivos. El descoser los hilos descubre incidentes donde la voz narradora es totalmente diferente de su madre y el prender con alfileres representa las similitudes entre la madre y la hija (Mencia, M y Husárová 2015, p. 40).

La obra finaliza, como señalaremos más adelante, con una secuencia generada al azar funcionando como metáfora de la relación entre la madre y la hija. Laura Borràs en el "Encontre d'escriptores" celebrado en Valencia en el 20IO establece un diálogo perfecto con el "saber oculto" de la tradición literaria femenina, con la metáfora del texto como "tejido" (De Gregorio 2OI2, p. 76). Por otro lado este trabajo invita al lector a tomar parte

\footnotetext{
${ }^{3}$ http://crissxross.net/elit/fitting_the_pattern.html
} 
en la exploración y a mediar en la construcción de las relaciones íntimas entre madre e hija. Nuestros estudiantes podrán adentrarse en el trabajo femenino de ser costurera, de hacerle a la voz narrativa y a su hermana vestidos a lo lardo de su historia y las consecuencias que les conllevaba a ellas y las relaciones que señalábamos anteriormente.

En la imagen de inicio se ofrece al lector las instrucciones para la lectura, así como algunas claves para poder captar la obra: es el patrón de memorias de la hija de una costurera. Se ofrece una tabla con las proporciones que corresponden a cada talla y, se informa de las medidas para el que está preparado el patrón, avisando de la necesidad de ajustarlas para la hija de la costurera cuya talla es inferior a la ofrecida.

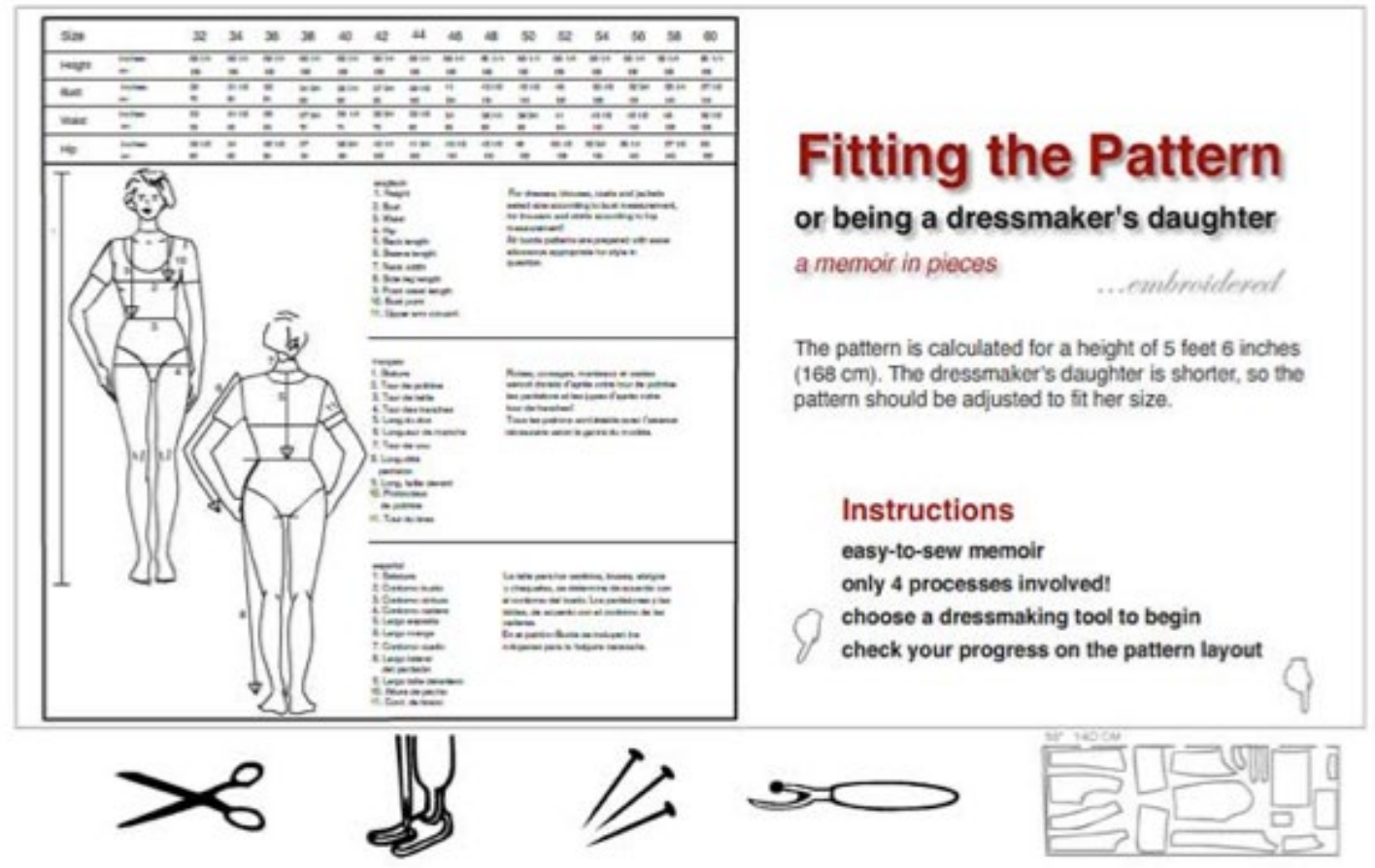

Ilustración 1 Inicio de Fitting the Pattern

Con esta presentación se crean una expectativas y unas orientaciones al lector, como la de llegar a concluir la obra con un traje final de recuerdos de la hija de la costurera. Este traje es presentado como un vestido, algo que puede esperarse a través de la sucesiva aparición de las palabras que aleatoriamente aparecen y desaparecen de la pantalla a modo de poema expresando la fuerza la complejidad y la naturaleza dinámica de la relación entre una madre y su hija. Junto con las palabras, las imágenes que se ven son la de una máquina de coser (la madre) que está cosiendo sobre un rollo de película (la hija de la costurera) en el que aparecen utensilios empleados en la confección de esta obra (lector) y sobre todo las palabras, que están relacionadas con lo narrado y con términos de costura y de filmación (profesión de la voz narrativa). Se fusionan el pasado y el presente, 
lo analógico y lo digital. Con este final se podría afirmar que la autora hace confluir en uno las diferentes piezas del patrón que ha ido creando el traje/memoria final (De Gregorio 2OI2, p. 80).
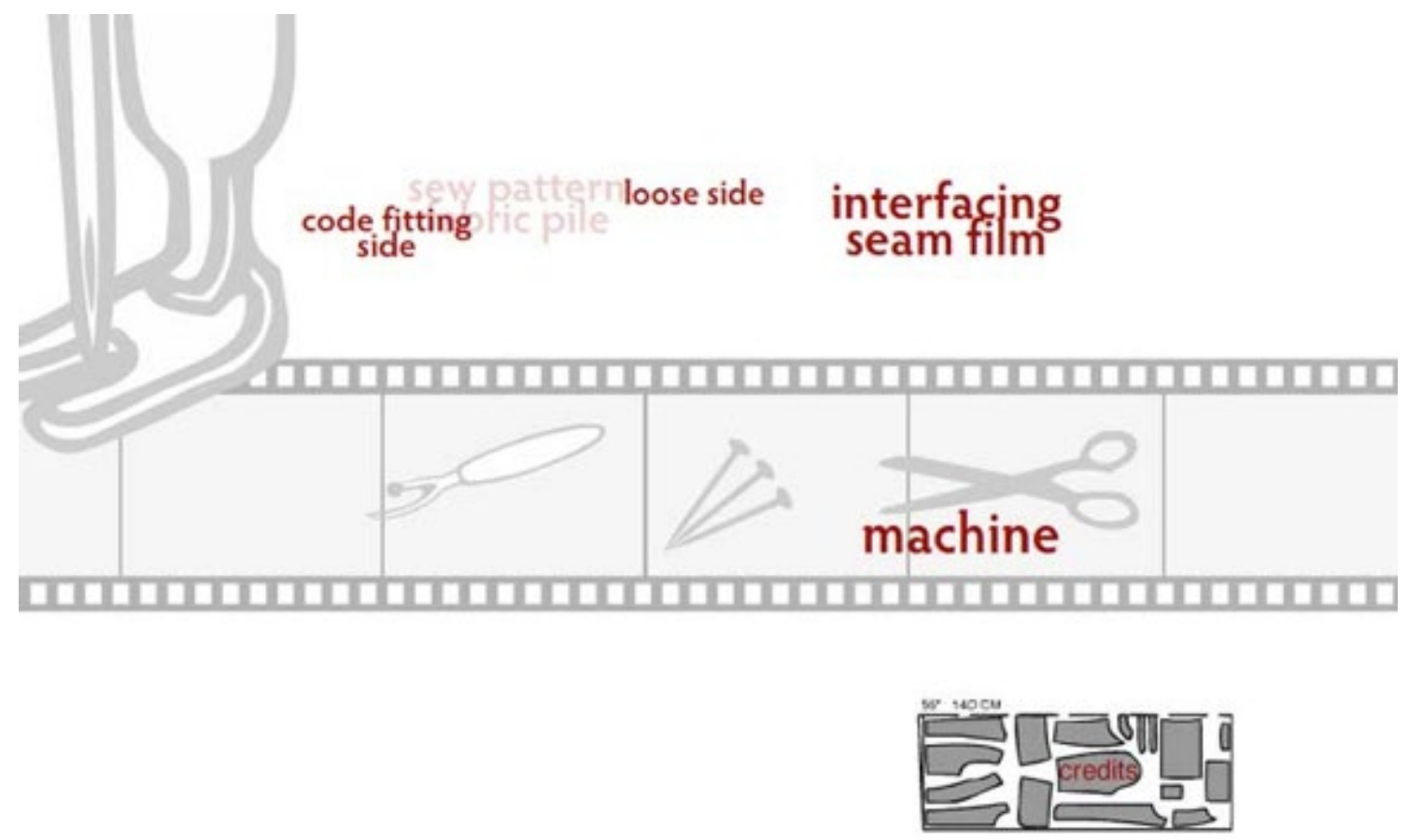

Ilustración 2 Final de Fitting the Pattern

Christine Wilks explica que con esta obra ha intentado reconvertir el oficio de su madre señalando que hay ciertos paralelismos entre el proceso creativo de ella y su propio proceso con los medios digitales por lo que tenía sentido utilizar patrones de costura en su interfaz gráfica de usuario. Estas similitudes y diferencias encajan en el medio digital y en el texto, literalmente sacado a través de la animación y dramatizada a través de la interactividad (Mencia, M y Husárová 20I5, p. 39).

Se ha ido nombrando alguno de los personajes que aparecen en la obra, pero cara a ayudar a nuestros estudiantes, sería conveniente que les hiciéramos una pequeña presentación de cada uno de ellos, que les ayudará a acercarse a ellos. El personaje principal es la narradora, es un personaje dinámico y redondo, por los numerosos rasgos que posee, por su capacidad de sorpresa y por los cambios que experimenta. Le sigue en importancia la madre, a quien la voz narradora va presentando hasta lograr que el lector se sienta a gusto y crezca una simpatía y un cariño hacia ella, es también un personaje dinámico y redondo. La tercera mujer que aparece, y tercer personaje es la hermana de la narradora. Es un personaje secundario del que poco se muestra, apenas se llega a saber que es escultura y que al igual que la voz narradora, de niña vestía los trajes realizados por su madre. Es pues, un personaje plano y estático. Finalmente el padre, personaje secundario, plano y 
estático, de él se puede decir que es un simple esbozo pues lo único que de él se recoge es que está enfermo de Alzheimer. A ningún personaje se le da la palabra de forma directa, solo sabemos aquello que la voz narrativa va dejándonos conocer excepto en una de las piezas del patrón en la que aparecen dos personas que son las únicas a las que la narradora les da la palabra, en los dos casos son personas anónimas que nos dan a conocer algún aspecto de la voz narrativa principal. Ninguno de los personajes es descrito físicamente, la información que se da está relacionada con su manera de ser, su profesión o como en el caso del padre, su enfermedad.

Uno de los aspectos que primero saltan a la vista al leer esta creación es que la elección de la sucesión de los acontecimientos corresponde al lector. Como ya se describió al inicio, el lector tiene la posibilidad de escoger uno de las cuatro herramientas de costura y posteriormente ir escogiendo los restantes. Una vez leídas las cuatro opciones, se vuelve a comenzar un ciclo nuevo, llegando a ofrecer cuatro. Por tanto, siempre hay que elegir cuatro elementos, pero es evidente que el orden que se escoja, va a adentrarnos y a mostrarnos la trama de una manera diferente y más sabiendo como se ha explicado al inicio de este apartado, el significado y el uso que cada herramienta otorga la autora.

Otro aspecto de la obra importante es el tiempo de la historia de Fitting the Pattern que comprende desde la infancia de la narradora hasta el momento presente, siempre en relación a su madre. El tiempo del discurso al estar configurado por la trama que es seleccionada en cierta medida por el lector con los instrumentos de costura, salta de una etapa de la vida de la voz narradora a otra. La historia vital del personaje lo vamos logrando a través de la voz narradora que con pequeños datos, como cuando iba de niña a la iglesia, las dificultades en su adolescencia, cuando estaba en la universidad... nos permiten ir estableciendo el tiempo de la historia que, lógicamente, como en toda narración, va a tener muchos espacios en blanco.

Para concluir estos rasgos generales de Fitting the Pattern citamos lo que la propia Wilks apunta:

As a part of a long-term tradition of exploring the imbrications between text and textile, Fitting the Pattern literalizes the idea that an act of writing is also an act of weaving. Here stiches are links, cloth fragments aldo textual fragments, and the reader is the tailor who must bring it all together to complete the pattern and make the narrative cohere.

Christine Wilks 2016 p. $4 \mathrm{I}$. 


\section{Aplicación práctica: leyendo Fitting the Pattern en el aula}

El acercamiento a la obra que se ha hecho en el apartado anterior permitirá que nuestros estudiantes puedan iniciar la obra con herramientas que le ayudan a comprender mejor la lectura. Además habrá que indicarles que han de superar la idea de la textualidad ligada a la palabra, para entender el texto como una unidad multimodal, en la que los significados utilizan diferentes códigos semióticos que deben ser descifrados en un todo significativo como se explicaba en el apartado anterior.

Junto con las dificultades de la lectura de obras digitales hay que añadir la dificultad del idioma pues, como se indicaba al principio del artículo, la propuesta es el uso Fitting the Pattern en el aula para estudiar tanto el idioma como de la cultura inglesa, en concreto el caso de dos generaciones de la mujeres británicas. Por tanto, hemos de ser conscientes que la competencia lingüística y lectora en inglés será menor que en su lengua materna y porque también es menor su conocimiento sobre la cultura, la sociedad, la retórica y las formas de vivir y comunicar (Valero, et al. 2OI5, p. 8).

Valero et al. (p. 9) señalan una serie de características de la lectura en línea en las lenguas extranjeras que puede aplicarse a la literatura digital y en este caso a la obra de Wilks. Para ellos lo primero que hay que destacar es un concepto que ya mencionamos anteriormente, la multimodalidad, que refleja cómo en los textos digitales se integran múltiples modos semióticos como vídeos, fotografías, diseños gráficos, etc. No son mera decoración sino que apoyan la comprensión. La segunda característica, la heterogeneidad textual e intertextualidad, en las obras no hay una uniformidad de formatos o estilos, al contrario diferentes textos se entremezclan de forma simultánea, dificultando el poder identificar las diversas voces. La tercera, la organización hipertextual con la que se pretende explicar cómo la red distribuye contenidos, recursos y materiales en piezas que se relacionan mediante enlaces, ventanas emergentes, textos dinámicos o piezas nuevas. Aunque no de forma específica para lectura de Fitting the Pattern pero sí como ayuda para la última característica está El control de la calidad de la información pues en la cultura de la participación, todos los internautas pueden agregar, manipular y comentar contenidos de otros usuarios. Finalmente, la multitarea, el estudiante a la vez que está leyendo la obra podrá emplear otras herramientas como diccionarios, traductores, páginas temáticas que le permitirán suplicar sus carencias.

Antes de iniciar la lectura, se tiene que tener en cuenta que un lector crítico es autónomo y consciente de que el significado de los textos es inseparable de las circunstancias históricas, culturales e institucionales en las que se generan todos los discursos, también las obras que se encuentran en Internet, son socioculturalmente situados y representan una visión del mundo determinada. (Valero et al. p. II y 2I). Por ello una de las actividades a realizar por nuestros estudiantes tras un primer contacto con la obra pasa por enseñarles a cuestionarse la posición que adopta la autora, la función del texto en su contexto, los valores contenidos, la selección de usos lingüísticos, los significados de los recursos que la autora emplea, los diseños gráficos, el sonido, etc. (Valero et al. p. 2I). 
Se propone a continuación un ejemplo de cómo sería un ejercicio a realizar en el aula con la obra, señalando los objetivos, la competencia, metodología y recursos necesarios, con alumnas y alumnos de $2^{\circ}$ año del grado de Estudios Ingleses.

Objetivos de X.

- Iniciar en el aprendizaje con la literatura digital.

- Capacitar para la lectura digital y ergódica.

- Capacitar para el aprendizaje para la navegación en Internet.

- Ejercitar una lectura crítica.

- Capacitar para el aprendizaje de elementos de la cultura inglesa.

- Familiarizar con características de la mujer inglesa.

\section{Competencias}

Conocimiento de los presupuestos fundamentales de la escritura crítica y lectura de la literatura.

Conocimiento de las costumbres y cultura inglesa actual.

Ser capaz de relacionar el conocimiento de las lenguas modernas con otras áreas y disciplinas.

Conocer de forma adecuada al nivel correspondiente la gramática, del léxico, los esquemas de organización textual de la lengua inglesa, la variación lingüística y la situación sociolingüística del inglés.

Ser capaz de buscar, tratar, sintetizar y difundir información de diversa índole de forma estructurada y sistemática haciendo uso de las distintas destrezas interpretativas de la disciplina.

Ser capaz de desarrollar estrategias de aprendizaje autónomo de gestión de la información.

Ser capaz de aplicar en la práctica los conocimientos adquiridos.

Ser capaz de trabajar en equipo a través dela presentación de ideas e información y de la negociación colectiva de soluciones.

\section{Recursos}

Un ordenador por cada dos estudiantes.

Auriculares para cada uno.

Metodología, desarrollo de la actividad

Número de alumnos: 30. Se proponen Io tareas para I5 parejas, por lo que las del II al I5 repetirán las cinco primeras.

Tres sesiones de una hora y cuarenta minutos. 


\section{Ia sesión.}

Se inicia con la explicación de qué es la literatura digital y sus principales características, para continuar con la presentación de Fitting the Pattern y Chritine Wilks. Se propone continuar antes de iniciar la lectura de la obra, con la lectura de las palabras de la autora para poder acercarse a la obra y a su contexto mejor:

I was born and brought up in the industrial West Riding of Yorkshire, the former heart of the English woolen textile industrym in Leeds and Morley, where generations of my family have live. Morley was a mill town, specialising in the production of shody cloth, which, coincidentally, is woven from repurposed fibres, shredded from old woollen garments and blankets. Leeds was the manufacturing birthplace of the ready-to-wear clothing industry and was also a centre of tailoring. Perhaps it was inevitable that my mother and her aprental grandmother had been employed in tailoring. Normally, in those days, women tailoring would only work one part of the garments (collars, butto.holes, sleeves, ... I'm guessing those were the things they worked on) but my great grandmother, being resourceful, kept asking to be put to work in diferente áreas. Eventually she worked her way around all the tailoring processes and learnt how to make a full suit.

(C. Wilks en M. Mencía y Z. Husárová, 2oI6, pp. 37-38)

Con la lectura anterior cada uno puede contextualizar a los personajes de la obra que va a leer, en el caso de tener dudas en la comprensión tanto en este momento como en cualquier otro del desarrollo de la actividad se les invita a consultar los diccionarios en red.

El tercer momento de la sesión será la división de los alumnos en parejas y las indicaciones para la tarea que tendrán que realizar.

Finalmente se terminará la sesión con una primera lectura de la obra. Es el momento para preguntar dudas, expresar sus dificultades ante la lectura de la misma, etc.

Concluimos pidiéndoles para la siguiente sesión la lectura completa completa de la obra.

\section{$2^{\text {a }}$ sesión}

Se le distribuye a cada pareja la tarea que tendrá que realizar en esa sesión y fuera del aula para en una tercera presentar al resto de la clase su aportación.

\section{Pareja I:}

La tarea de la primera pareja conssitirá en buscar e informarse sobre la sociedad trabajadora inglesa del siglo XX en el ámbito textil, la descripción y el análisis de Leeds y Morley como centro de la industria textil inglesa. 


\section{Pareja 2:}

Tendrán que profundizar en las cuatro mujeres, describiendo el contexto social de cada una y si ha habido una progresión en las generaciones. Además, tendrán que analizar las consecuencias sociales y económicas que tenía en las mujeres y en las familias el que éstas solo aprendiesen a coser una parte de las piezas del traje, tanto por lo que se refleje en la obra como lo que investiguen.

\section{Pareja 3:}

El tercer grupo podrá centrarse en los registros que aparecen en la narrativa, las expresiones que se emplean, si estas reflejan un grupo social, si son inglés estándar, o lenguaje coloquial, etc.

\section{Pareja 4:}

Tendrán que realizar una línea temporal de los diferentes textos que van surgiendo para ayudarnos a conocer mejor a las mujeres que aparecen y que el grupo i ha profundizado.

\section{Pareja 5:}

En la narración se nos presenta los trabajos de las hijas de la modista, los estudiantes analizarán la evolución socio-laboral de los personajes.

\section{Pareja 6:}

El sexto grupo buscará los principales campos semánticos y realizará con ello un glosario bilingüe.

\section{Pareja 7:}

Comoles hemos explicado en nuestra presentación (sesión I) de la obra, los miembros de las restantes parejas han de buscar los diferentes sentimientos que las distintas herramientas muestran. Así, este grupo ha de analizar los sentimientos en relación con la madre que se muestran en la maquina de coser (muestran los sentimientos diferentes a la madre).

\section{Pareja 8:}

Los miembros de este grupo tendrán que analizar los sentimientos que se extraen de las tijeras (son las emociones negativas).

\section{Pareja 9:}

En este grupo la herramienta a analizar es el descosedor.

\section{Pareja Io:}

La última pareja tendrá que hacerlo con los alfileres. 


\section{$3^{\text {a Sesión }}$}

En esta última sesión cada pareja presentará su trabajo, que se habrá ido tutorizando a través del campus virtual y de las tutorías. Con la comunicación de cada grupo se irá construyendo, analizando y comentando Fitting the Pattern.

Aunque la lectura de la obra requiere mucho tiempo y esfuerzo, así como las tareas a realizar, la respuesta de los estudiantes es interesante, en un primer momento se sienten muy atraídos por la obra y empiezan a navegar e ir de un lado a otro cosiendo, cortando, descosiendo... sin apenas leer. Después, al intentar ahondar en el texto, se chocan no solo con la dificultad del idioma, sino el que éste surja aleatoriamente dificultándoles la compresión de la trama. Como se señaló anteriormente, Wilks estaba interesada en que hasta el final no se "terminase el traje", no se completasen sus memorias, de ahí la dificultad del lector ante la obra y aún más en otro idioma. Y, así ante su propia experimentación, pondrán experimentar lo que ya les habíamos explicado sobre por qué este tipo de literatura se define como ergódica, término que Espen Aarseth apropió del mundo de la física y que proviene de los vocablos griegos ergon y hodos que significan "obra/trabajo" y "camino", de ahí la literatura ergódica es aquella creación literaria que exige un esfuerzo no trivial por parte del lector para poder atravesar, transitar por el texto, penetrar en su sentido (Sánchez- Mesa, 2004: II8). El empleo de la literatura digital en el aula puede considerarse también una tarea ergódica, pero es precisamente el esfuerzo, el medio, la calidad de la obra lo que posibilita que la experiencia sea positiva.

Con todo ello, el objetivo inicial de utilizar la literatura digital para realizar actividades de lectura en la adquisición de un segundo idioma, con el acercamiento al estudio de elementos de la cultura inglesa y las características de la mujer de la sociedad británica, son muy positivas. 


\section{Referencias bibliográficas}

-Aarseth, E. (2004). La literatura ergódica. en Sánchez- Mesa (ed.). Literatura y Cibercultura, Madrid: Arco/libros, págs. II7-I45.

- Borràs, L. Memmott, T., Raley, R. Stefans, B. (2OII). Electronic Literatire Collection, Volumen Two. Electronic Literature Organization. Obtenido desde http://collection.eliterature.org/2/

-Del-Moral, Ma E., Villalustre, L. y Neira, Ma R. (20I6). Relatos digitales: activando las competencias comunicativa, narrativa y digital en la formación inicial del profesorado. $O c$ nos, I5 (I), 22-4I. Obtenido el 20 de octubre de 2018 en https://revista.uclm.es/index.php/ ocnos/article/view/ocnos_2Or6.I5.I.923.

- Gervais, B. (2007). Reading in a Era of Hipertextuality. En R. Siemens y S. Schreibiman (ed.) A Companion to Digital Literary Studies (pp. I83-202). Malden, Ma: Blackwell Publishing.

- Goicoechea de Jorge, M. (2OII). Los nuevos rituales de lectura literaria en pantalla. Encuentros en Verines, Encuentro XXVII “La literatura en la Era Digital”, Obtenido en octubre 2018 desde http://www.mcu.es/lectura/libroSearch.do?action=busquedaInicial\& params. anio $=2$ oII\&cache $=$ init\&layout $=$ verines.

- Hayles, N. K. (2008), Electronic Literature. New Horizonsfor Literary, Indiana: University of Notre Dame.

- Hayles, N. K. (20I0), How We Read: Close, Gyoerm Mahine, en ADE Bulletin I5o (I8) ( pp. 62-79). Obtenido en septiembre 2018 desde https://www.ade.mla.org/content/down$\mathrm{load} / 79 \mathrm{I} 5 / 225678$.

- Mencía, M., Husárová, Z. (eds.) (2015). Creative Manual for Repurposing in Electronic L9945iterature. Kosice, Slovakia : Dive Buki.

- Montesa, S. (ed.) Literatura e Internet. Nuevos textos, nuevos lectores, ( pp. 385-402). AEDILE.

- Morales Sánchez, I. (2OII), El lector itinerante: nuevos espacios, nuevos retos. En S. Montesa (ed) Literatura e Internet. Nuevos textos. Nuevos lectores (pp. 387-402). AEDILE Obtenido en febrero 2019 desde http://www.cervantesvirtual.com/obra/literaturae-internet--nuevos-textos-nuevos-lectores/?_ga=2.9725046r.3287849.1554975205IO9284937.I554975205. 
- Morales Sánchez, I. (2018) . Leer literatura en la era digital, en Palabra Clave (La Plata), 7(2): eo49. Disponible el 8 de febrero de 2019 en: http://www.memoria.fahce.unlp.edu.ar/ art_revistas/pr.8534/pr.8534.pdf.

- Morales Sánchez, I. (2OI9). Inmersiones lectoras. Literatura y lectura digital. En José Cordón García (Coord.) Libro, lectores y lectura digital. Metodologías humanísticas en la era digital. Alicante: Instituto Juan Andrés de Comparatística y Globalización.

- Pressman, J. (20I4). Digital Modernism. Making It New in New Media. New York: Oxford University Press.

- Torres-Toukoumidis, A., Romero-Rodríguez, L., Pérez-Rodríguez, M. A., \& Björk, S. (20i6). Desarrollo de habilidades de lectura a través de los videojuegos: Estado del arte. Ocnos, I5 (2), 37-49. Obtenido el 20 de octubre de 2018 en https://revista.uclm.es/index. php/ocnos/article/view/ocnos_2OI6.I5.2.II24.

- Valero, M. J., Vázquez, B. y Cassany, D. (2015). Desenredando la web: la lectura crítica de los aprendices de lenguas extranjeras en entornos digitales. Ocnos, I3, 7-23. Obtenido el 20 de octubre de 2018 en https://revista.uclm.es/index.php/ocnos/article/view/ocnos_2OI5.I3.OI 